\title{
Caspase activation pathways: some recent progress
}

\author{
SP Cullen ${ }^{1}$ and SJ Martin ${ }^{\star 1}$ \\ Cell Death and Differentiation (2009) 16, 935-938; doi:10.1038/cdd.2009.59
}

Three major pathways to apoptosis-associated caspase activation have been identified in mammals: the extrinsic or death receptor pathway, the intrinsic or apoptosome pathway and the cytotoxic lymphocyte-initiated granzyme B pathway. ${ }^{1}$ The order of events in each of these pathways to caspase activation is by now relatively well understood. However, recent studies including one published in this issue of $C D D^{2}$, have generated new insights into caspase activation pathways in response to signals that provoke cell damage, as well as in contexts in which caspase activation does not result in apoptosis.

\section{Refinement of the apoptosome-initiated caspase activation cascade}

The order of caspase activation events within the apoptosome-initiated caspase activation pathway was originally defined using cell-free systems. ${ }^{3-6}$ In this pathway, release of mitochondrial cytochrome $c$ as a consequence of opening of the Bax/Bak channel results in the assembly of the Apaf-1/ caspase- 9 apoptosome and activation of caspase- 9 within this complex. ${ }^{3}$ Using immunodepleting monoclonal antibodies to remove individual caspases from cell-free extracts, it was found that caspase-9 propagates a cascade of further caspase processing events by directly cleaving and activating caspase-3 and caspase-7.,6 Caspase-3 then processes caspases- 6 and -2 , and in turn, caspase- 6 processes caspases-8 and -10 downstream (Figure 1). Although this order of events has been partly validated using MEFs deficient in caspase-3 and caspase-7, it remained unclear whether the precise order of caspase activation events in this pathway is representative of what happens in a cellular context.

Using a selective inhibiter of caspase-3 (M-791), as well as siRNA-mediated ablation of specific caspases, Inoue et al have now validated the order of caspase activation events within the apoptosome pathway and have also refined this cascade by detecting earlier unobserved redundancy between caspases-3 and -7 with respect to the processing of caspase- 6 and -2 . $^{2}$ The authors used the specific inhibitor of caspase- 3 to discriminate between the ability of caspase- 3 and -7 to propagate Apaf-1-dependent caspase activation in response to various cytotoxic drugs. They found that whereas the poly-caspase inhibitor zVADfmk blocked all caspase processing downstream of caspase-9, or the specific caspase-3 inhibitor was only effective in blocking the processing of caspase-3, while processing of caspases $-2,-6,-7$ and -8 was largely unaffected.

On the basis of the existing cascade (Figure 1), the expected outcome of caspase-3 inhibition within the apoptosome pathway would be inhibition of caspase $-2,-6$, and -8 processing, as these are known to occur as a consequence of caspase- 3 activation. This suggested that another caspase was capable of substituting for caspase-3, with the obvious candidate being caspase-7. The authors subsequently used CASP-3-deficient MCF-7 cells, together with siRNA-mediated ablation of caspase-7, to show that caspase-7 does indeed substitute for the loss of caspase- 3 by processing caspases -2 and -6 , thus enabling active caspase -6 to process caspase- 8 downstream. ${ }^{2}$ Although these data confirm that the hierarchy of caspase activation within the intrinsic pathway to caspase activation conforms to that delineated using cell-free systems, they also highlight earlier unappreciated redundancy between caspases -3 and -7 with respect to processing of the other caspases activated within this pathway (Figure 1).

\section{Caspases-3 and -7 do not process the same repertoire of substrates}

However, although caspases -3 and -7 show partial redundancy with respect to processing of caspases -2 and -6 , these effector caspases are not fully redundant with respect to many other substrates. Using normalized amounts of caspases-3 and -7 , it has been found that caspase-3 shows greater activity towards many of the substrates that are cleaved during the terminal or demolition phase of apoptosis. ${ }^{7}$ For example, at equimolar levels, caspase-3 cleaves Bid, gelsolin and RIP1 much more efficently than caspase-7. By contrast, both caspases cleave fodrin, RhoGDI2 and PARP with equal efficiency and co-chaperone p23 is a preferred substrate for caspase-7, ${ }^{7}$ an observation also confirmed by Inoue et al. ${ }^{2}$ Hence, although caspases -3 and -7 have overlapping substrate preferences, these proteases are non-redundant and do show distinct activity towards certain substrates. These observations go some way towards explaining why $C A S P-3$ and $C A S P-7$ null mice show distinct phenotypes on some genetic backgrounds. ${ }^{8}$ In this regard, a recent study has also highlighted differences between CASP-3 and CASP-7 null mice in terms of resistance to endotoxin-induced lethality, with the latter animals showing much greater resistance to endotoxin treatment than the former. ${ }^{9}$ The underlying

\footnotetext{
${ }^{1}$ Molecular Cell Biology Laboratory, Department of Genetics, Trinity College, The Smurfit Institute, Dublin 2, Ireland

*Corresponding author: SJ Martin, Molecular Cell Biology Laboratory, Department of Genetics, Trinity College Dublin, The Smurfit Institute of Genetics, Dublin D2, Ireland. Tel: + 353189612 89; Fax: + 353167985 58; E-mail: martinsj@tcd.ie
} 


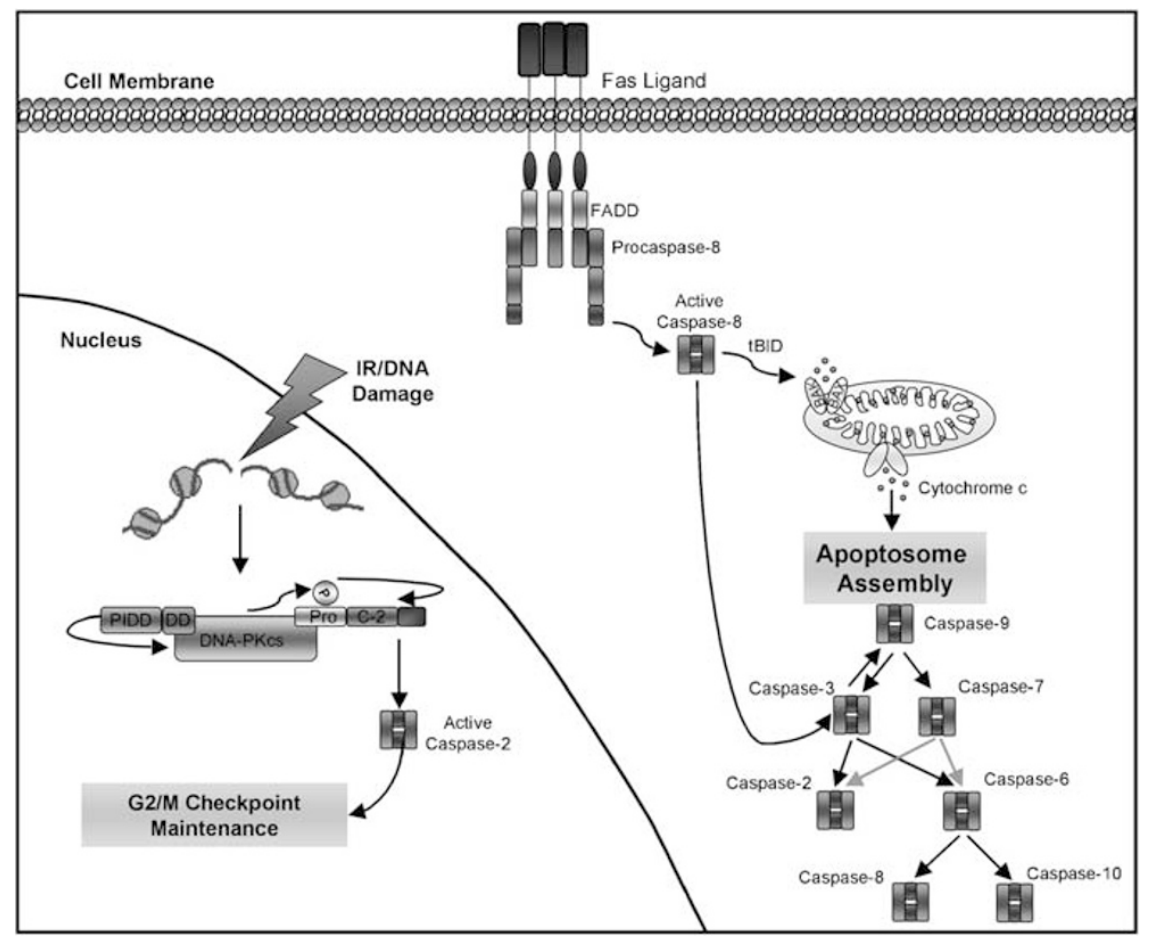

Figure 1 Selected caspase activation pathways. Three pathways to caspase activation are illustrated. Binding of Fas ligand to Fas promotes oligomerization of caspase-8 through the adaptor molecule FADD. In type I cells, activated caspase-8 then activates caspase-3 through direct proteolytic processing, leading to further downstream caspase activation and substrate proteolysis. Caspase-8 can also initiate the mitochondrial pathway to apoptosis by cleaving the BH3-only protein, BID. Cleaved BID promotes BAX/ BAK-dependent cytochrome $c$ release from mitochondria and formation of the apoptosome, resulting in activation of caspase-9. Active caspase-9 then instigates a caspase activation cascade by processing caspases -3 and -7 , which propagate further caspase processing events, as depicted. As described by Inoue et al in this issue of CDD, ${ }^{2}$ caspase-7 can propagate further caspase activation events in this cascade when caspase- 3 is absent or disabled. A new pathway to caspase-2 activation in response to DNA damage has recently been described that leads to cell-cycle arrest rather than apoptosis. In this pathway, DNA damage leads to activation of DNA-PKcs which phosphorylates and activates caspase-2. The kinase activity of DNA-PKcs can be enhanced by PIDD which can be found in a tripartite complex with DNA-Pkcs and caspase-2. Active caspase-2 then promotes a G2/M DNA-damage checkpoint through a mechanism that is not understood at present

molecular mechanism for this observed difference is unknown but might be related to another recent observation which suggests that caspase-7, unlike caspase- 3 , is proteolytically processed and activated by caspase- $1 .{ }^{10}$ Thus, caspase-7 may be a downstream effector for caspase-1-dependent proinflammatory effects in some circumstances.

\section{Caspase-2 activation and DNA damage-associated checkpoint control}

The role of caspase- 2 in cell death cascades has long been puzzling. ${ }^{11}$ Although caspase-2 is essentially always processed during apoptosis, mice lacking the CASP-2 gene fail to show any significant impairments in cell-death control. ${ }^{12,13}$ Furthermore, it is also intriguing that while caspase-2 has the structure of an initiator caspase - possessing a long prodomain containing a CARD motif - it is really rather poor at proteolytically processing other caspases. Moreover, as already discussed above, caspase-2 is proteolytically processed and activated by caspase- 3 within the apoptosome caspase activation cascade, which would place this caspase as a downstream effector rather than an upstream initiator of caspase activation. ${ }^{5}$
Recent studies now suggest a completely new role for caspase-2 in the context of DNA damage-associated cellcycle arrest which may well turn out to be the primary function of this caspase. ${ }^{14,15}$ Several groups have earlier reported that DNA damaging agents, such as etoposide, can promote early activation of caspase-2, upstream of cytochrome $c$ release. ${ }^{16-18}$ What has been controversial is whether early caspase-2 activation promotes cytochrome $c$ release in the intrinsic pathway, as caspase inhibitors have little effect on the latter event. This makes it unlikely that caspase-2 is an important instigator of mitochondrial cytochrome $c$ release within the intrinsic pathway. However, Shi et al have now found that caspase-2 can form a complex within the nucleus with the DNA damage-related kinase DNA-PKcs. ${ }^{14}$ On activation by DNA damage, DNA-PKcs promotes phosphorylation of caspase-2 and this phosphorylation event seems to drive caspase-2 processing. However, processing of caspase-2 in this context does not result in apoptosis, and cells deficient in this caspase do not show any impairment in DNA damage-associated apoptosis. ${ }^{14}$ When cells suffer DNA damage during the cell cycle, the typical response seen is cell-cycle arrest to allow for DNA repair. Cells damaged during S or G2 typically arrest at the G2/M boundary, however Shi et al found that CASP-2 null cells fail to show this G2/M arrest, 
showing a greater percentage of mitotic cells than wild-type cells in response to DNA damage. The ataxia telangiectasia mutant (ATM) kinase plays a prominent role in regulating the G2/M checkpoint in response to double-stranded DNA breaks. However, the requirement for caspase-2 in G2/M arrest was largely independent of ATM activity, suggesting the involvment of another kinase. The authors also showed a role for PIDD in facilitating the kinase activity of DNA-PKcs, which in turn mediates caspase-2 phosphorylation leading to G2/M cell-cycle arrest in response to DNA damage. ${ }^{14}$

The identity of the substrate(s) cleaved by caspase- 2 in response to DNA damage remain unknown. One possible target is Bid, as this BH3-only protein has earlier been implicated in a DNA-damage-response pathway and is phosphorylated by the DNA damage responsive kinase ATM. ${ }^{19,20}$ BID null animals show a defective DNA-damage checkpoint similar to what has been observed in CASP-2 null mice. As Bid is one of the few substrates that has been found for caspase-2, it is tempting to speculate that proteolysis of Bid in the context of DNA damage contributes to the caspase2-dependent DNA damage checkpoint. How cleaved Bid may promote cell-cycle arrest remains completely obscure however.

A very recent study by Kumar et al also suggests that caspase-2 plays an important role in cell-cycle transtitions. ${ }^{15}$ They report that $C A S P-2$ null cells proliferate faster, are more readily transformed by Ras/E1A oncogenes and also form more aggressive tumors in a Myc-driven tumor model. Thus, a role for caspase-2 as a tumor suppressor protein that contributes to cell-cycle control does seem very likely.

\section{Caspase-8 and monocyte-macrophage differentiation}

The extrinsic pathway to caspase activation is initiated through ligation of membrane death receptors, which recruits the cytosolic adapter protein, FADD, and caspase-8, which form the death-inducing signaling complex (DISC). ${ }^{21,22}$ On joining the DISC, caspase- 8 is activated and promotes apoptosis by activating downstream effector caspases either directly or indirectly. The role of caspase-8 in death receptorinduced apoptosis has been well described, however, recent evidence suggests that this caspase may play a role in some important non-apoptotic processes.

Caspase- 8 is critical for embryonic development as mice deficient in this caspase die in utero with accumulated defects in the heart and neural tube and show deficiencies in hemopoietic progenitor cell production. ${ }^{23,24}$ As this phenotype is markedly different to loss of death receptors such as TNF, this suggests a death receptor-independent function for caspase-8. A link between caspases and differentiation had been proposed as it was observed that blocking caspase activation through the use of various caspase inhibitors prevented differentiation of monocytes into macrophages. ${ }^{25}$ Furthermore, deletion of CASP-8 in macrophage precursors using Cre/loxP recombination compromises their differentiation to macrophages. ${ }^{26}$ Surprisingly, caspase- 8 activation in this context does not require death receptor ligation or result in apoptosis, suggesting a death receptor-independent, nonapoptotic role for this initiator caspase in macrophage differentiation. ${ }^{25}$ Despite the proteomic identification of a number of caspase substrates that are cleaved during this process, ${ }^{27}$ the precise role of caspase- 8 in promoting differentiation of monocytes to macrophages has remained unclear until recently.

Solary et al have now shown a role for caspase-8-mediated proteolysis of RIP1 in macrophage differentiation. ${ }^{28}$ Exposure of monocytes to M-CSF promotes the formation of a complex comprising caspase-8, FADD, FLIP and RIP1, which facilitates caspase- 8 activation and RIP1 proteolysis. Cleaved RIP1 then down-regulates NF- $\kappa$ B activity, basal levels of which may favor macrophage differentiation. ${ }^{28}$ However, overexpression of caspase-8-resistant RIP1 only partially impeded this process suggesting that other caspase- 8 targets play a role here. ${ }^{28}$ Dynamic cytoskeletal changes occur during differentiation and cell adhesion also plays an important role in this context. In this regard, several cytoskeletal and adhesion proteins have been identified as caspase- 8 substrates during macrophage differentiation, which suggests that caspase-8 may target multiple factors to propagate this process. ${ }^{27}$ It is interesting that caspase- 8 activity has also been shown to be required for the differentiation of human placental villous trophoblast cells, ${ }^{29}$ suggesting that a role for caspase-8 in differentiation may not be restricted to the monocyte/macrophage lineage.

\section{Concluding remarks}

As Inoue et al have illustrated in this issue, ${ }^{2}$ caspases are activated through proteolytic cascades, which can amplify an initially small amount of caspase activity to levels sufficient for the initiation of apoptosis. As certain caspases are efficient instigators of cell death, it goes without saying that activation of this subset of caspases during non-apoptotic processes must be tightly controlled to prevent the balance of caspase activation irreversibly tipping towards apoptosis. However, it is exactly this aspect of caspase regulation that is very poorly understood at present. As we have outlined in this commentary, several lines of evidence now argue that caspases normally activated during apoptosis can undergo limited activation during disparate processes with endpoints other than cell death. How this is achieved still remains to be determined.

\footnotetext{
1. Taylor RC, Cullen SP, Martin SJ. Nat Rev Mol Cell Biol 2008; 9: 231-241

2. Inoue S, Browne G, Cohen GM. Cell Death Differ 2009; 16: 1053-1061.

3. Li $\mathrm{P}$ et al. Cell 1997; 91: 479-489.

4. Srinivasula SM, Ahmad M, Fernandes-Alnemri T, Alnemri ES. Mol Cell 1998; 1: 949-957.

5. Slee EA, Adrain C, Martin SJ. J Biol Chem 2001; 276: 7320-7326.

6. Slee EA et al. J Cell Biol 1999; 144: 281-292.

7. Walsh JG et al. Proc Natl Acad Sci USA 2008; 105: 12815-12819.

8. Lakhani SA et al. Science 2006; 311: 847-851.

9. Lamkanfi M et al. Blood 2009; 113: 2742-2745

10. Lamkanfi M et al. Mol Cell Proteomics 2008; 7: 2350-2363.

11. Krumschnabel $\mathrm{G}$ et al. Cell Death Differ 2009; 16: 195-207.

12. Bergeron L et al. Genes Dev 1998; 12: 1304-1314.

13. O'Reilly LA et al. Cell Death Differ 2002; 9: 832-841.

14. Shi $\mathrm{M}$ et al. Cell 2009; 136: 508-520.

15. Ho LH et al. Proc Natl Acad Sci USA 2009; 106: 5336-5341.

16. Robertson JD et al. J Biol Chem 2002; 277: 29803-29809.

17. Lassus P, Opitz-Araya X, Lazebnik $Y$ et al. Science 2002; 297: 1352-1354.
} 
24. Sakamaki K et al. Cell Death Differ 2002; 9: 1196-1206

18. Lin CF et al. J Biol Chem 2004; $279: 40755-40761$.

25. Sordet $O$ et al. Blood 2002; 100: 4446-4453.

19. Kamer I et al. Cell 2005; 122: 593-603.

20. Zinkel SS et al. Cell 2005; 122: 579-591.

21. Nagata S. Biddable death. Nat Cell Biol 1999; 1: 143-145.

22. Peter ME, Krammer PH. Cell Death Differ 2003; 10: 26-35.

23. Varfolomeev EE et al. Immunity 1998; 9: 267-276.

26. Kang TB et al. J Immunol 2004; 173: 2976-2984.

27. Cathelin S et al. J Biol Chem 2006; 281: 17779-17788.

28. Rebe $C$ et al. Blood 2007; 109: 1442-1450.

29. Black S. Cell Death Differ 2004; 11: 90-98. 